Western University

Scholarship@Western

$11-15-2021$

\title{
Interventions for preventing relapse or recurrence of major depressive disorder in adults in a primary care setting: a network meta-analysis
}

\author{
Andrew S. Moriarty \\ University of York \\ Lindsay Robertson \\ University of York \\ Faraz Mughal \\ Keele University, School of Medicine \\ Natalie Cook \\ Tees Esk and Wear Valleys NHS Foundation Trust \\ Simon Gilbody \\ University of York
}

See next page for additional authors

Follow this and additional works at: https://ir.lib.uwo.ca/epidempub

Citation of this paper:

Moriarty, Andrew S.; Robertson, Lindsay; Mughal, Faraz; Cook, Natalie; Gilbody, Simon; McMillan, Dean; Chew-Graham, Carolyn A.; Ali, Shehzad; Hetrick, Sarah E.; Churchill, Rachel; and Meader, Nicholas, "Interventions for preventing relapse or recurrence of major depressive disorder in adults in a primary care setting: a network meta-analysis" (2021). Epidemiology and Biostatistics Publications. 167.

https://ir.lib.uwo.ca/epidempub/167 


\section{Authors}

Andrew S. Moriarty, Lindsay Robertson, Faraz Mughal, Natalie Cook, Simon Gilbody, Dean McMillan, Carolyn A. Chew-Graham, Shehzad Ali, Sarah E. Hetrick, Rachel Churchill, and Nicholas Meader 


\section{UNIVERSITY of York}

This is a repository copy of Interventions for preventing relapse or recurrence of major depressive disorder in adults in a primary care setting: a network meta-analysis.

White Rose Research Online URL for this paper:

https://eprints.whiterose.ac.uk/180650/

Version: Published Version

\section{Article:}

Moriarty, Andrew orcid.org/0000-0003-0770-3262, Robertson, Lindsay, Mughal, Faraz et al. (8 more authors) (2021) Interventions for preventing relapse or recurrence of major depressive disorder in adults in a primary care setting: a network meta-analysis. Cochrane Database of Systematic Reviews. ISSN 1469-493X

https://doi.org/10.1002/14651858.CD014832/full

\section{Reuse}

Items deposited in White Rose Research Online are protected by copyright, with all rights reserved unless indicated otherwise. They may be downloaded and/or printed for private study, or other acts as permitted by national copyright laws. The publisher or other rights holders may allow further reproduction and re-use of the full text version. This is indicated by the licence information on the White Rose Research Online record for the item.

\section{Takedown}

If you consider content in White Rose Research Online to be in breach of UK law, please notify us by emailing eprints@whiterose.ac.uk including the URL of the record and the reason for the withdrawal request. 


\section{Interventions for preventing relapse or recurrence of major depressive disorder in adults in a primary care setting: a network meta-analysis (Protocol)}

Moriarty AS, Robertson L, Mughal F, Cook N, Gilbody S, McMillan D, Chew-Graham CA, Ali S, Hetrick SE, Churchill R, Meader N

Moriarty AS, Robertson L, Mughal F, Cook N, Gilbody S, McMillan D, Chew-Graham CA, Ali S, Hetrick SE, Churchill R, Meader N. Interventions for preventing relapse or recurrence of major depressive disorder in adults in a primary care setting: a network meta-analysis (Protocol).

Cochrane Database of Systematic Reviews 2021, Issue 11. Art. No.: CD014832.

DOI: 10.1002/14651858.CD014832. 
TABLE OF CONTENTS

ABSTRACT 1

BACKGROUND

OBJECTIVES

4

METHODS

4

ACKNOWLEDGEMENTS

REFERENCES

APPENDICES

CONTRIBUTIONS OF AUTHORS

14

DECLARATIONS OF INTEREST

SOURCES OF SUPPORT 
[Intervention Protocol]

\section{Interventions for preventing relapse or recurrence of major depressive disorder in adults in a primary care setting: a network meta-analysis}

Andrew S Moriarty1,2, Lindsay Robertson 3 ,4, Faraz Mughal5,6, Natalie Cook7, Simon Gilbody1,2, Dean McMillan¹, Carolyn A ChewGraham $^{5}$, Shehzad Ali1 ${ }^{1}$, Sarah E Hetrick ${ }^{8,9}$, Rachel Churchill 3,4 , Nicholas Meader 3,4

1Mental Health and Addiction Research Group, Department of Health Sciences, University of York, York, UK. ${ }^{2 H u l l ~ Y o r k ~ M e d i c a l ~ S c h o o l, ~}$ University of York, York, UK. ${ }^{3}$ Cochrane Common Mental Disorders, University of York, York, UK. ${ }^{4}$ Centre for Reviews and Dissemination, University of York, York, UK. ${ }^{5}$ School of Medicine, Keele University, Keele, UK. 6 Unit of Academic Primary Care, Warwick Medical School, University of Warwick, Coventry, UK. ${ }^{7}$ Tees, Esk and Wear Valleys NHS Foundation Trust, York, UK. ${ }^{8}$ Department of Psychological Medicine, Faculty of Medical and Health Sciences, The University of Auckland, Auckland, New Zealand. ${ }^{9}$ Children and Young People Satellite, Cochrane Common Mental Disorders, The University of Auckland, Auckland, New Zealand

Contact: Andrew S Moriarty, andrew.moriarty@york.ac.uk.

Editorial group: Cochrane Common Mental Disorders Group.

Publication status and date: New, published in Issue 11, 2021.

Citation: Moriarty AS, Robertson L, Mughal F, Cook N, Gilbody S, McMillan D, Chew-Graham CA, Ali S, Hetrick SE, Churchill R, Meader N. Interventions for preventing relapse or recurrence of major depressive disorder in adults in a primary care setting: a network metaanalysis (Protocol). Cochrane Database of Systematic Reviews 2021, Issue 11. Art. No.: CD014832. DOI: 10.1002/14651858.CD014832.

Copyright @ 2021 The Cochrane Collaboration. Published by John Wiley \& Sons, Ltd.

\section{A B S T R A C T}

\section{Objectives}

This is a protocol for a Cochrane Review (intervention). The objectives are as follows:

The aim is to assess, using network meta-analysis (NMA), the effectiveness and safety of interventions for preventing relapse-recurrence of depression in adults in a primary care setting. NMA is a technique for comparing three or more interventions simultaneously in a single analysis by combining both direct and indirect evidence across a network of studies.

The objectives are to:

1. estimate the relative effectiveness of pharmacological, psychological and other interventions to prevent relapse-recurrence, compared with each other and controls (e.g. pill placebo, treatment as usual) on relapse-recurrence outcomes and adverse events;

2. assess the effect of interventions on quality of life outcomes and social and occupational functioning;

3. estimate the relative ranking of included interventions on relapse-recurrence, quality of life, and adverse events;

4. summarise availability and principal findings of eligible economic evaluations. 


\section{B A C K G R O U N D}

\section{Description of the condition}

Depression is now the leading cause of disability worldwide (WHO 2017), with a prevalence in excess of 264 million people globally (GBD 2017). It results in significant morbidity for patients and exerts a high societal and economic cost (Richards 2011). Rather than being a phenotypically homogenous condition (i.e. presenting in the same way in all people who experience depression), clinical presentation and trajectories vary across patients. Some people with depression experience a single, time-limited episode, and no further episodes beyond that (Monroe 2011). Depression for others, however, is a recurrent condition, with patients experiencing the reemergence (relapse or recurrence) of depressive symptoms after a period of relative wellness (Beshai 2011). There is some evidence to suggest that the risk of relapse and recurrence increases with a greater number of depressive episodes experienced, but decreases as the period of recovery gets longer (Beshai 2011; Solomon 2000). The economic burden of depression is also significantly higher in those who experience a relapse or recurrence compared to those who do not (Gauthier 2019). Therefore, intervening to prevent relapse and recurrence of depression is likely to improve the overall course of illness for individual patients.

Relapse and recurrence of depression are usually defined with respect to three further terms: response, remission and recovery. According to most recent recommendations (Frank 1991; Rush 2006), response is an initial improvement of symptoms, usually after treatment initiation and usually attributable to the treatment. After three weeks of minimal symptoms, a patient can be said to have entered remission. Any subsequent re-emergence of depressive symptoms after this point is described as a relapse (a return to the index episode of depression). If a relapse has not occurred by four months after remission, a patient is said to have entered recovery, after which point any re-emergence of depressive symptoms is termed a recurrence (a new episode of depression separate from the index episode).

The original description of relapse, recurrence and the other change-points by Frank 1991 presented a consensus view of how these terms should be used to facilitate a more consistent application in research. There is also probably a meaningful clinical distinction to be made between a relapse back to the index episode of depression before full recovery and the recurrence of depression in the form of a new and discrete depressive episode, following recovery. Subsequent efforts to validate the dichotomy (or contrast between the two terms) have been few, but are broadly supportive of the distinction (Beshai 2011; Riso 1997), although the precise temporal cut-offs have not been empirically derived and remain inconsistently operationalised in the literature (Bockting 2015). Therefore, for the purpose of this review, we will use the term 'relapse-recurrence', which has been used in previous reviews of interventions (Cox 2012; Vittengl 2007), to mitigate for inconsistencies in how the terms are applied in the included studies.

The majority of work exploring the scope of the problem of depressive relapse-recurrence has been done in secondary care settings and is likely to be of limited applicability to primary care (Buckman 2018), which is where the vast majority of patients with depression are managed (Rait 2009). Relapse-recurrence rates, and longer-term outcomes generally, tend to be worse in speciality settings, compared to primary care settings (Ormel 2020). One primary care cohort study that followed up people with remitted depression found that $37.1 \%$ of participants relapsed within one year (Lin 1998). It is worth noting that this study was relatively small $(n=251)$ and followed up participants from randomised trials (all of whom had been prescribed antidepressant medication); therefore, this study's findings are potentially not generalisable to all primary care populations. A more recent UK-based cohort study followed up patients who had received low-intensity cognitive behavioural therapy (CBT) through the Improving Access to Psychological Therapies (IAPT) service of the UK National Health Service NHS. Conducted in a community care setting, this study found that $53 \%$ of participants experienced a relapse-recurrence within one year, and that the majority $(79 \%)$ of those participants did so within the first six months (Ali 2017). This study is also not necessarily representative of a typical primary care population, as not all patients with depression in primary care would be referred to the IAPT service.

Potentially more useful information can be gained from nonclinical, naturalistic cohort studies. Two systematic reviews have examined studies with naturalistic cohorts (Steinert 2014; Van Weel-Baumgarten 2000). One review estimated primary care relapse-recurrence rates to be between $30 \%$ and $40 \%$ (Van WeelBaumgarten 2000). The other review found that $35 \%$ to $60 \%$ of participants experienced stable recovery (Steinert 2014). A study of trajectories of depression in primary care suggests that the majority of patients with depression in primary care follow a mild trajectory of illness (Gunn 2013).

In addition to patients who experience single or recurrent episodes of depression, there is a further group of patients who have a more chronic and persistent form of depression. An estimate from a review of naturalistic cohorts suggests that between $10 \%$ and $17 \%$ of depressed patients in primary care follow a chronic rather than episodic course of illness (Steinert 2014). The concepts of relapse and recurrence are less easily applied to these patients, although this group of patients can include those with recurrent depression, with incomplete remission between episodes. Indeed, Monroe 2011 cautioned against excluding patients with chronic or persistent depression from studies of relapse and recurrence; the majority of these patients do in fact still remit or recover at times and can therefore be said to experience a relapse and recurrence, despite the longer duration of index episodes. In summary, the cohort of patients seen in primary care is different from those in secondary care, and the studies discussed here have limitations that prohibit us from making definitive statements about relapse-recurrence rates in primary care settings.

\section{Description of the intervention}

There are three recognised treatment phases for depression: treatments implemented before any symptomatic improvement, with a view to achieving remission (acute phase); those employed after symptomatic improvement but before recovery (continuation phase); and those that extend past the point of recovery (maintenance phase) (Bockting 2015). Interventions to prevent relapse-recurrence might be targeted at patients who are in the continuation or maintenance phases, having had symptomatic improvement; or might be implemented during the acute phase, with the intention of exerting a protective effect against relapse or recurrence in the future (Bockting 2015). 
Pharmacological and psychological interventions are comparable in terms of efficacy for acute phase depression (Cuijpers 2013; Kamenov 2016), and combination therapy is superior to either type of intervention given singly (Kamenov 2016). Like the acute phase interventions, interventions for preventing relapse-recurrence of depression can broadly be considered pharmacological (i.e. antidepressant medications (ADMs)) or psychological. Other kinds of non-pharmacological therapies might include social interventions (befriending, financial support), service-level interventions (for example, collaborative care), and combination interventions. Physical interventions such as electroconvulsive treatment (ECT) can be used to prevent relapse in some patients, although ECT is almost exclusively a secondary care intervention, evidence for its efficacy is of low quality and there are concerns around harm caused by ECT (NICE 2018).

There is reasonable evidence that compared with placebo, ADMs prevent depression relapse-recurrence; there do not appear to be major differences in this effect across different ADMs, or even different classes of ADMs (Geddes 2003; Glue 2010; Hansen 2008; Kaymaz 2008). A recent systematic review supported the preexisting evidence that compared to placebo, continuation of ADMs for at least six months after remission significantly improved relapse-recurrence rates, and that this was true for selective serotonin reuptake inhibitors (SSRIs), tricyclic antidepressants (TCAs) and other newer agents (Kato 2020). Absolute benefits from ADMs are greater for those at higher initial risk of relapserecurrence (Geddes 2003), and the effect may decrease after a number of depressive episodes, as patients appear to develop resistance to their prophylactic properties (Kaymaz 2008). There are some limitations in the available literature around the effect of ADMs on relapse-recurrence, which prevent us from drawing firm conclusions about their effects in primary care populations. The studies included in existing reviews have all been undertaken in secondary care populations, where outcomes are generally poorer; the included studies, on the whole, have not adjusted for potential withdrawal effects upon stopping ADMs, which are likely to be a significant confounding factor when estimating rates of relapserecurrence (Cohen 2010; Hengartner 2020; Recalt 2019). ADMs are also not without their drawbacks:

- adverse effects are common (including sleep disturbance, gastrointestinal disturbance and sexual dysfunction, among others) (Van Leeuwen 2020), and can be worse in elderly people (Coupland 2011);

- concerns about dependence and withdrawal symptoms (which can often be confused with relapse, although these generally occur much sooner after ADM discontinuation than relapse, and can be severe and long-lasting (Davies 2019; Van Leeuwen 2020));

- reluctance by patients to take medication;

- poor concordance (i.e. not taking the medication as prescribed or recommended); and

- high financial cost to the health service (Maund 2019).

The other issue with ADMs is that any prophylactic or protective effects disappear when the medication is discontinued (Bockting 2015), whereas the effect from some types of psychological therapy, like CBT and problem-solving therapy (PST), can endure beyond the point at which the therapy is actually being administered (Cuijpers 2013; Vittengl 2007).
Psychological therapies for preventing relapse-recurrence include CBT, mindfulness-based cognitive therapy (MBCT), PST, and interpersonal therapy (IPT) (Clarke 2015). CBT aims to modify thoughts and behaviours, such as reducing avoidance, increasing activities and challenging negative thoughts. CBT also involves the teaching of cognitive skills which focus on challenging underlying dysfunctional beliefs (cognitive content) that can persist after remission or recovery despite a non-depressed state, presenting a vulnerability that might be more easily triggered by, for example, life events or stress (Beshai 2011; Bockting 2015; Clarke 2015). MBCT was developed specifically as an intervention to prevent relapserecurrence (Kuyken 2015) and, in addition to cognitive content, also focuses on cognitive processes. MBCT teaches patients to experience thoughts without judgement and to recognise that negative thoughts are transient and do not have to guide feelings or behaviours. IPT focuses on interpersonal and societal role problems, which can be implicated in the onset and recurrence of depressive symptoms (Clarke 2015).

The UK National Institute for Health and Care Excellence (NICE) currently recommend that patients starting ADM for depression should continue treatment for a minimum of six months after remission to reduce the risk of relapse. People "at risk of relapse" are advised to continue ADM for a minimum of two years (this includes people who have had two or more episodes of depression, have residual symptoms, have a history of severe or prolonged episodes or inadequate response, or for whom "the consequences of relapse are likely to be severe"). Recommendations for relapse prevention psychological therapies are: individual CBT for people who have relapsed despite ADM, or for people with a significant history of depression and residual symptoms despite treatment; and MBCT for patients who have had three episodes or more of depression (NICE 2009). In more severe cases, patients are usually referred for specialist treatment where relapse prevention interventions can include further high-intensity psychological treatment, lithium augmentation of ADM and ECT. The Canadian Network for Mood and Anxiety Treatments (CANMAT) depression guideline concurs with NICE that evidence to support maintenance therapy for longer than two years is less wellestablished, but that certain risk factors (early depression onset, ongoing psychosocial adversity, older age, and comorbid medical or psychiatric conditions) might justify extended maintenance ADM treatment (Lam 2009). The American Psychiatric Association (APA) guideline recommends continuation treatment with ADM and depression-focussed psychological treatments. For those with three or more previous depressive episodes, chronic depression or other risk factors (including family history of mood disorder), the APA advises maintenance treatment with the ADM treatment that produced and maintained remission during the acute and continuation phases, and for ECT to be considered beyond that. They recommend regular monitoring for "signs of relapse" throughout (Van Kempen 2010).

\section{How the intervention might work}

One route by which interventions for preventing relapserecurrence might work is through modifying neural pathways; ADMs primarily exert their pharmacological effects on the seratonergic, dopaminergic and noradrenergic pathways (Healy 1997). A comprehensive overview of these neurobiological processes is beyond the scope of this review, but one mechanism by which ADMs are thought to prevent relapse-recurrence through this 
route is by reducing the presence of residual depressive symptoms (those that are sub-threshold and persist after remission), which are strongly associated with increased risk of relapse-recurrence (Buckman 2018; Lin 1998). While neurotransmitters are likely to be implicated in some patients with depression, and modification of these pathways is a sound theoretical basis for explaining some of the effectiveness of ADMs at preventing relapse-recurrence, it is widely accepted that depression is multifactorial. Indeed, a recent study reports that for this reason, doctors increasingly avoid giving overly simplistic biological explanations to patients (Tickell 2020).

Psychological therapies exert their effect by modifying a broader range of therapeutic targets. Most psychological therapies for preventing relapse-recurrence are designed to be provided during the continuation or maintenance treatment phases; although, as discussed, these can be given during the acute phase and exert a longer-term benefit. As well as aiming to reduce residual depressive symptoms (like ADMs) psychological therapies additionally target cognitive and information processing mechanisms (specifically those involved in integrating affective and cognitive information; processing negatively valenced stimuli; social skills and the ability to use social support; problem solving skills; and degree of negative self-concept) and interpersonal stress pathways. Relapse of depression is associated with negative thinking styles, such as rumination (Buckman 2018). CBT specifically targets these thoughts and aims to educate patients on how to modify and transform such thoughts into more positive thoughts (Clarke 2015). $\mathrm{MBCT}$ focusses on teaching patients to improve their awareness of and relationship to such thoughts, rather than on modifying the thoughts (Kuyken 2015). Psychological therapies also focus on being aware of and planning for early warning signs of relapse-recurrence, and also focus on healthy lifestyle behaviours. In summary, there are a range of mechanisms by which the interventions eligible for inclusion in this review might work to improve relapse-recurrence outcomes.

\section{Why it is important to do this review}

We have discussed the burden associated with depression in terms of patient morbidity and quality of life, as well as the wider cost to society as a whole. Despite scientific progress and an increased understanding of the biological and psychological underpinnings of mental health conditions, there is no evidence of a decrease in morbidity or mortality from depression, in contrast to physical health conditions such as cardiovascular disease, stroke and cancer (Insel 2009). Furthermore, the vast majority of people with depression are managed in primary care (Ramanuj 2019), yet the majority of research on depressive relapse-recurrence has been undertaken in a secondary care context. There is a need for feasible and scalable interventions that can be implemented in a primary care clinical setting and, by better understanding what interventions and intervention components work best, this review could inform such efforts.

Other Cochrane Reviews have looked at similar interventions and outcomes. One review assessed the effectiveness of continuation and maintenance treatments for persistent depressive disorder, mainly in a secondary care context (Machmutow 2019), and found that the paucity and moderate/high risk of bias in the available studies meant it was uncertain whether pharmacological or psychological therapies were effective for preventing relapserecurrence.There has been a Cochrane Review of relapse prevention interventions in children and adolescents (Cox
2012) and an ongoing review is exploring the effectiveness of interventions aimed at teaching patients to recognise early warning signs (EWS) of depression to prevent recurrence (Lenora 2019). These will form part of a larger body of evidence looking at relapse prevention more generally.

There has been only one previous systematic review of relapse prevention strategies, specifically in a primary care setting (Gili 2015); this review identified only three studies. There have been a number of new primary care-based RCTs of relapse prevention interventions since the publication of that review. A synthesis of available evidence is needed to guide the allocation of interventions in primary care to prevent relapse-recurrence and improve disease trajectories for patients. As we have seen, it is not well understood which interventions work best for which patients, and national guidelines differ in terms of risk stratification and treatment recommendations. This speaks to the fact that depression is complex and centred in a biopsychosocial context. Furthermore, there are multiple kinds of interventions available. Patient preferences and individual patient circumstances will always be important in guiding treatment decisions through discussion with healthcare professionals. An ability to be able to inform patients about the relative effectiveness of the range of different treatment options is an essential part of this decisionmaking process. This review aims to provide a robust summary of the available evidence to support such discussions.

\section{O B JECT IVES}

The aim is to assess, using network meta-analysis (NMA), the effectiveness and safety of interventions for preventing relapserecurrence of depression in adults in a primary care setting. NMA is a technique for comparing three or more interventions simultaneously in a single analysis by combining both direct and indirect evidence across a network of studies.

The objectives are to:

1. estimate the relative effectiveness of pharmacological, psychological and other interventions to prevent relapserecurrence, compared with each other and controls (e.g. pill placebo, treatment as usual) on relapse-recurrence outcomes and adverse events;

2. assess the effect of interventions on quality of life outcomes and social and occupational functioning;

3. estimate the relative ranking of included interventions on relapse-recurrence, quality of life, and adverse events;

4. summarise availability and principal findings of eligible economic evaluations.

\section{METHODS}

\section{Criteria for considering studies for this review}

\section{Types of studies}

We will include all RCTs (including cluster- and cross-over RCTs) that include direct comparisons between two or more eligible interventions and/or usual care, control or placebo. The latter are useful for NMA, as placebo can be a common comparator and therefore can be an important component of a network of interventions. All studies must have taken place in a primary care or community setting. A primary care setting is the first 
point of contact for a patient accessing health services (e.g in a general practitioner's clinic) and interventions are usually delivered by a generalist. By community settings, we mean interventions delivered outside of a traditional primary care setting (e.g. those delivered in community centres or community clinics) but not requiring a specialist referral to a secondary care setting. Community settings include nursing and residential care settings, as long as there is no specialist or secondary care involvement. Services providing first-line psychological treatments (for example, the UK's IAPT service (Clark 2011)) are also often termed community rather than primary care, and their cohorts are likely to be directly applicable to a primary care setting. These will be all included, but setting (primary or community care) will be explored as a potential source of heterogeneity.

\section{Types of participants}

Adults (age 18 years and over) with major depressive disorder (MDD), diagnosed in line with Diagnostic and Statistical Manual of Mental Disorders (DSM-V) criteria, either through diagnostic interview or using a validated diagnostic questionnaire, who have entered remission or recovery before receiving the intervention. Participants with remission or recovery would be defined as those patients who had met diagnostic criteria for MDD at baseline but no longer met these diagnostic criteria. In this review, we will not include interventions delivered during the acute phase of depression. It is likely that the majority, if not all, of the included studies will have tested interventions in participants with recurrent depression.

We will not include participants with severe mental illness (for example, bipolar affective disorder or schizophrenia), as these participants would not represent a population comparable with those with only MDD and therefore would be a potential threat to the transivity assumption (similarity of the direct and indirect evidence) of the network meta-analyses. We will include participants with other psychological co-morbidities which are routinely managed in primary care (for example, anxiety, posttraumatic stress disorder, obsessive-compulsive disorder). We will also include participants with physical co-morbidities and longterm conditions, and will explore this as a potential source of heterogeneity if appropriate to assess whether this may contribute to intransitivity. In addition, criteria for comparing the similarity of participant characteristics of included studies is outlined in "Assessment of clinical and methodological heterogeneity within treatment comparisons" below.

\section{Types of interventions}

RCTs of any intervention specifically aimed at preventing relapse or recurrence of depression in a primary or community care setting. These could be pharmacological, psychological or social (e.g. collaborative care, community outreach, befriending). We expect that some RCTs may compare different interventions (e.g. psychological versus pharmacological approach) rather than a control arm. As discussed, physical therapies such as ECT are not relevant to a primary care setting and therefore will be excluded from this review.

The following are interventions of clinical relevance (decision-set interventions).

- Pharmacological interventions: Previous reviews have not suggested significant differences in the effectiveness of different
ADMs on relapse and recurrence across primary and secondary care settings, but there may be a dose-related effect (Hansen 2008). Where different doses or drug classes have been trialled, we will merge classes (e.g. SSRI, TCA, serotonin-norepinephrine reuptake inhibitor (SNRI)) and will split these by dose (low, medium and high strength), according to equivalent doses per the British National Formularly.

- Psychological interventions will be categorised by type (for example: CBT, MBCT, IPT), and intensity (for example, highversus low-intensity CBT, as defined by the authors of primary studies). Group and individual psychological interventions will be treated a separate nodes in the NMA.

- Other interventions: as discussed, interventions that aim to prevent relapse-recurrence, but which are not either pharmacological or psychological will be included, as long as they meet the other inclusion criteria.

The following are supplementary-set interventions and are included to give greater power and to connect up the network, but are not themselves of clinical relevance:

- Placebo, control, or 'treatment as usual' arms. These can be defined as deemed appropriate by the trial authors. We will extract information about the content of these arms to ensure that they are directly comparable.

\section{Types of outcome measures}

\section{Primary outcomes}

- Time to relapse-recurrence

Time to relapse-recurrence provides a more detailed picture of intervention effectiveness than a binary outcome (i.e. relapserecurrence or no relapse-recurrence). We will define participants with relapse-recurrence as those who had previously met the definition for remission or recovery but subsequently met the diagnostic criteria for MDD.

The primary harm outcome is adverse events (for example, discontinuation due to side effects, self-harm, suicide, or hospitalisation).

\section{Secondary outcomes}

- Binary relapse-recurrence outcome

- Antidepressant medication dose changes (change in dose after intervention, to enable us to assess whether interventions led to increased or decreased ADM prescription)

- Quality of life measures (e.g. EQ-5D, SF-36, or any other instrument)

- Social and occupational functioning (e.g. Global Assessment of Function Scale (GAF), Occupational Functioning Scale (OFS), or any other instrument)

\section{Timing of outcome assessment}

We will analyse outcome data at the following time points: shortterm (less than six months), medium-term (six to 12 months) or long-term (greater than 12 months).

\section{Hierarchy of outcome measures}

Due to the great likelihood of more than one reported eligible outcome, we will include data as per the following rules. 
- In case of available data from both observer-rating scales and self-report questionnaires, we will prioritise data from observer-rating scales. For the remission/recovery and relapserecurrence outcomes, we will give preference to diagnostic interview or HAM-D and for the self-reporting scale, we will give preference to the PHQ-9 or BDI.

- In case of several outcome measures of the same hierarchy level used in one trial, we will select the outcome measure most frequently used across all trials.

- In case of several outcome measures of the same hierarchy level and the same availability across trials, we will randomly select the outcome measure.

- Where applicable, if diagnostic interview, HAM-D or BDI were not reported, we will select the outcome measure most frequently used across all trials.

\section{Economic commentary}

We will develop a brief economic commentary based on current methods guidelines (Aluko 2020) to summarise the availability and principal findings of trial-based full economic evaluations (costeffectiveness analyses, cost-utility analyses, cost-benefit analyses) that compare psychological or pharmacological relapse prevention interventions with each other or with controls for depression. This commentary will focus on the extent to which principal findings of eligible economic evaluations indicate that an intervention is judged favourably (or unfavourably) from an economic perspective, when implemented in different settings. A separate search will be conducted to identify economic evidence, including: screening the reference lists and conducting forward citation tracking from eligible studies of effects identified for inclusion in the main review; a search of NHS Economic Evaluation Database (NHS EED); and the use of specialist search filters to sets of records retrieved by searches of one or two selected general electronic biomedical literature databases searched for the main review of intervention effects (as recommended by the Cochrane Economics Methods Group).

Identified economic evaluations will be screened against the inclusion criteria by one review author (ASM). Data will be extracted including: basic details of the characteristics of each identified economic evaluation (including analytic framework and type of economic evaluation, analytic perspective and time horizon, main cost items included in each analysis, and setting); and brief text extracts that summarise their principal findings (verbatim text on conclusions drawn by the authors of each economic evaluation and text that summarises uncertainty surrounding authors' principal conclusions).

The findings of the brief economic commentary will be incorporated into the 'Discussion' section and will include a brief narrative summary of: the electronic health economics literature databases searched; the number of relevant economic evaluations identified for each eligible comparison (each eligible intervention/ comparison combination); the descriptive information collected from each study; principal conclusions as reported by the authors of each analysis (with respect to the base case analysis); and principal sources of uncertainty regarding authors' principal conclusions (based on the results of any sensitivity analyses conducted).

\section{Search methods for identification of studies}

\section{Electronic searches}

An Information Specialist with the Cochrane Common Mental Disorders Group (CCMD) will run searches on the following bibliographic databases and trial registers using relevant subject headings (controlled vocabularies) and search syntax, appropriate to each resource.

- Cochrane Common Mental Disorders Controlled Trials Register (CCMDCTR) (all available years).

- The Cochrane Central Register of Controlled Trials (CENTRAL) (the Cochrane Library, latest issue).

- Ovid MEDLINE (1946 to date) (Appendix 1).

- Ovid Embase (1974 to date).

- Ovid PsycINFO (all available years).

- ISI Web of Science: Conference Proceedings Citation IndexScience (CPCl-S) (1990 to date).

- ClinicalTrials.gov (www.clinicaltrials.gov) (all available years).

- World Health Organization International Clinical Trials Registry Platform (apps.who.int/trialsearch/) (all available years).

There will be no restrictions on date, language or publication status applied to the searches.

\section{Searching other resources}

The Information Specialist will also search the following sources of grey literature (primarily for dissertations and theses).

- Open Grey (www.opengrey.eu).

- ProQuest Dissertations \& Theses Global (www.proquest.com/ products-services/pqdtglobal.html).

- DART-Europe E-theses Portal (www.dart-europe.eu).

- EThOS - the British Libraries e-theses online service (ethos.bl.uk).

- Open Access Theses and Dissertations (oatd.org).

\section{Reference lists}

We will check the reference lists of all included articles and relevant systematic reviews to identify additional research missed from the original electronic searches (e.g. ongoing studies, unpublished or in-press citations).

\section{Personal communication}

We will contact authors and subject experts for information on unpublished or ongoing studies, or to request additional data.

\section{Data collection and analysis}

\section{Selection of studies}

All abstracts will be independently screened by two review authors (from among AM, LR, FM, NC), with any disagreements to be referred to a third review author (NM). The same process will apply to full-text screening. We will identify and exclude duplicate records and we will collate multiple reports that relate to the same study, so that each study, rather than each report, is the unit of interest in the review. We will record the selection process in sufficient detail to present an adapted PRISMA flow chart of study selection and 'Characteristics of excluded studies' table. 


\section{Data extraction and management}

We will extract data using a pre-prepared and pre-piloted data collection form. Two review authors (from among ASM, LR, FM, NC) will independently extract data, and any disagreements or discrepancies will be resolved by referral to a third review author (NM). We will extract the following study intervention and population characteristics that may act as effect modifiers:

- methods: study design, total duration of study, number of study centres and location, study setting (primary care, community), withdrawals, and date of study;

- participants: number, mean age, age range, gender, baseline severity of condition, diagnostic criteria, number of previous episodes, length of index episode, recurrence status, anxiety symptoms; inclusion criteria, and exclusion criteria, suiciderelated behaviours; level of suicide ideation; risk of suicide; comorbid chronic physical illness, psychiatric co-morbidity; socioeconomic status;

- interventions: type and duration of acute phase intervention; type of relapse-recurrence prevention intervention; comparison; dose (including intended and actual dose received); duration of treatment; concomitant medications; concomitant psychosocial interventions (including type, duration, who delivered the intervention, whether it was delivered to groups or individuals, was manualised, actual number of sessions attended); and excluded medications;

- outcomes: primary and secondary outcomes specified and collected, and time points reported;

- notes: funding for trial, and notable conflicts of interest of trial authors.

\section{Outcome data}

We will extract from each included study arm-level data about relapse-recurrence rates and time to relapse-recurrence, the duration of the intervention, the number of participants, the drop-out rates, the authors' definition of relapse-recurrence, the number of patients that experienced a relapse-recurrence and the interventions being compared. When extracting data about type and duration of acute phase interventions, we will also extract information about how the individual studies have controlled for withdrawal confounding (Hengartner 2020).

\section{Other data}

We will extract and report a narrative summary of any costeffectiveness analyses taking place alongside the RCT. If outcome data are not reported in a usable way (e.g. missing standard deviations (SD) or $95 \%$ confidence intervals (Cls)), we will note this in the 'Characteristics of included studies' table. We will resolve disagreements by consensus or by involving a third review author (NM). One review author (ASM) will transfer data into the Review Manager 5.4 file (Review Manager 2020) or RevMan Web (RevMan Web 2019). We will double-check that data are entered correctly by comparing the data presented in our review with the study reports. A second review author (from LR, FM or NC) will spot-check study characteristics for accuracy against the study report.

\section{Assessment of risk of bias in included studies}

Two review authors (ASM, LR, FM, NC) working independently will undertake risk of bias assessment, using version 2 of the Cochrane risk of bias tool for randomised controlled trials (RoB 2) (Higgins
2021; Sterne 2019), with respect to effect of assignment. We will do this for the two primary outcomes (time to relapse-recurrence within 12 months; adverse outcomes at any timepoint). A third review author will resolve any disagreements. We will use the methods described in the ROB 2 guidance for Cluster RCTs. We will assess the risk of bias according to the following domains:

- random sequence generation;

- allocation concealment;

- blinding of participants and personnel;

- blinding of outcome assessment;

- incomplete outcome data;

- selective outcome reporting;

- other bias.

We will judge each potential source of bias as either high, low or unclear, and will provide a supporting quotation from the study report together with a justification for our judgment in the 'Risk of bias' table. We will summarise the 'Risk of bias' judgements across different studies for each of the domains listed. We will use the RoB2 excel tool to manage assessments and will store documentation of judgements and assessments on a data repository website for scientific data, where they will be available to readers of the full review. Where necessary, we will contact the trial authors for further information. Where information on risk of bias relates to unpublished data or correspondence with a trial author, we will note this in the 'Risk of bias' table. We will present all 'Risk of bias' data graphically and in the text.

\section{Measures of treatment effect}

\section{Relative treatment effect}

Where time to relapse-recurrence has been assessed, we will use hazard ratios (HRs). We will express dichotomous outcome data as risk ratios (RRs) with $95 \% \mathrm{Cls}$. We will express continuous data as standardised mean differences (SMD) with $95 \% \mathrm{Cls}$. To aid interpretation of SMDs we will back-transform estimates to the most commonly reported scale for that outcome. Results from the NMA will be presented as summary relative effect sizes (SMD or RR) for each possible pair of treatments.

\section{Relative treatment ranking}

Where appropriate, we will also estimate the ranking probabilities for all treatments of being the most effective treatment using $P$ values, which take into account effect estimates and precision (Chaimani 2020). However, if we identify too much uncertainty we will not rank treatments.

\section{Unit of analysis issues}

\section{Cluster-randomised trials}

Cluster-RCTs involve the randomizsation of participants as groups (or clusters) rather than as individuals. If we include cluster-RCTs, but reported data have not been appropriately adjusted for the correlation between participant outcomes within clusters, we will contact trial authors to obtain an estimate of the intra-cluster correlation coefficient (ICC); or impute the ICC using estimates from the other included trials, or from similar external trials. We will inflate the reported standard errors by the square root of the design effect, using the estimated/imputed ICC (Higgins 2020). We 
will undertake sensitivity analyses to assess the robustness of the combined intervention effects to assumptions regarding the ICCs.

\section{Cross-over trials}

If we include cross-over trials (trials where participants receive a sequence of different interventions), we will only use data from the first cross-over period, due to the risk of carry-over effects.

\section{Studies with multiple treatment groups}

For multi-arm trials, we will use graph-theoretical methods (using the R software package 'netmeta', R) to account for between-arm correlations. The netmeta package treats multi-arm trials as a complete subgraph with known variances. For each comparison within the trial, variances are back-calculated adjusting for correlation between arms (Rucker 2012).

\section{Dealing with missing data}

We will attempt to obtain any missing data (data necessary for our analysis that is not reported in the trial paper) from study authors. If we are unable to obtain missing SDs from study authors, we will estimate these from $\mathrm{P}$ values, t-statistics, $\mathrm{Cls}$ or standard errors, if reported in the paper.

If a vast majority of SDs are available and only a minority of SDs are unavailable or unobtainable, we plan to use the method devised by Furukawa and colleagues to impute SDs and calculate percentage responders (Furukawa 2005; Furukawa 2006). If we use this method, we will interpret data with caution and will take into account the degree of observed heterogeneity. We will also undertake a sensitivity analysis to examine the effect of the decision to use imputed data.

If additional figures are not available or obtainable and it is not deemed appropriate to use the Furukawa method as described above, we will not include the trial data in the comparison of interest.

We will pool 'last observation carried forward' (LOCF) data and other data from other imputation methods, such as multiple imputation or mixed effect models (rather than mix LOCF and observed case (OC) data). We will also undertake sensitivity analyses using OC data, where available.

\section{Assessment of heterogeneity}

\section{Assessment of clinical and methodological heterogeneity within treatment comparisons}

Heterogeneity and transitivity are related. Heterogeneity results from variation of effect modifiers within a treatment comparison, while intransitivity results from variation of effect modifiers across treatment comparisons (Efthimiou 2016). To evaluate the presence of clinical heterogeneity we will generate and compare descriptive statistics for trial and study population characteristics across all eligible trials that compare each pair of interventions.

When we find heterogeneity, we will attempt to determine potential reasons for it by examining individual study and subgroup characteristics. We expect the following characteristics to potentially introduce clinical heterogeneity and intransitivity.

- Study setting (primary care or community).

- Type of depression (first-episode; recurrent; chronic).
- Age of participants.

- Comorbid anxiety.

- Physical comorbidity (long-term conditions).

- Gender.

- Socio-economic status.

- Length of follow-up/timing of relapse.

- Dose of treatment (e.g. milligram dosage of ADMs, length and number of sessions with for psychological interventions).

\section{Assessment of statistical heterogeneity}

Assumptions when estimating the heterogeneity

In NMAs, we will assume a common estimate for the heterogeneity variance across the different comparisons.

\section{Measures and tests for heterogeneity}

We will assess statistical heterogeneity using the 12 statistic and its $95 \% \mathrm{Cl}$ and $\mathrm{Tau}^{2}$ (between-study variance). ${ }^{2}$ quantifies heterogeneity across studies by measuring the percentage of variability that cannot be attributed to random error. The Cochrane Handbook for Systematic Reviews of Interventions provides the following rule of thumb for interpreting $\left.\right|^{2}$ values (Deeks 2020):

- $0 \%$ to $40 \%$ : might not be important;

- 30\% to 60\%: may represent moderate heterogeneity;

- $50 \%$ to $90 \%$ : may represent substantial heterogeneity;

- $75 \%$ to $100 \%$ : considerable heterogeneity.

The $1^{2}$ will be interpreted in light of its magnitude and direction of effects and the strength of evidence for heterogeneity (width of $95 \%$ $\mathrm{Cl})$.

\section{Assessment of transitivity across treatment comparisons}

As described, transitivity assessment is related to heterogeneity assessment, with transitivity being specifically applicable to NMA and relating to differences in the distribution of different effect modifiers across different comparisons. We will assess transitivity by comparing the distribution of effect modifiers across the different comparisons for each outcome.

\section{Assessment of statistical inconsistency}

Inconsistency (also called incoherence) is the statistical manifestation of intransitivity in the data. Inconsistency occurs when direct and indirect evidence for a particular relative effect are in disagreement, and is only possible to detect when there are evidence loops (sources of direct and indirect evidence for the same comparison).

\section{Local approaches for evaluating inconsistency}

Local approaches focus on a particular comparison and usually involve assessing the absolute difference between the two summary estimates in the form of an inconsistency factor (IF). We will use a loop-specific approach (a loop of evidence is formed when at least three pairs of treatments have been compared and form a closed path). An IF will be calculated by contrasting direct estimates with indirect estimates. The magnitude of the IFs, their $95 \% \mathrm{Cls}$ and a loop-specific Z-test will be used to infer about the presence of inconsistency in each loop. We will extend analysis to all closed loops assuming a loop-specific heterogeneity and examine 
the estimates of inconsistency together with 95\% Cls for each loop, using a graphical representation. We will assume a common heterogeneity estimate within each loop and will be cautious deriving conclusions from this approach. Netmeta software also provides pairwise meta-analyses estimates as part of the analyses and we will informally compare these estimates with the results found in our network meta-analyses.

\section{Global approaches for evaluating inconsistency}

We will also perform a global assessment of inconsistency to check the assumption of consistency in the entire network. We will model inconsistency using the design-by-treatment interaction model (Higgins 2012), which accounts for different source of inconsistency that can occur when studies with different designs (two-arm trials versus three-arm trials) give different results as well as disagreement between direct and indirect evidence.

\section{Assessment of reporting biases}

We will use a framework described in the Cochrane Handbook (Page 2019) that proposes an assessment be made for each comparison regarding the risk and potential impact of missing data from studies ("known-unknowns") and risk of missing studies ("unknownunknowns"). We will use this framework in our GRADE assessments to guide our judgements on 'undetected' or 'suspected' reporting bias.

For our primary outcome, we will be guided by our risk of bias assessments, as well as by comparison-adjusted funnel plots (extensions of funnel plots for use in network meta-analyses), if there are a minimum of ten studies (Chaimani 2012). Where there is evidence of funnel plot asymmetry, we will adjust for small-study effects using WinBUGS/OpenBUGS (Mavridis 2016).

\section{Data synthesis}

Assuming that we have a connected network of treatments, we will perform NMAs using the netmeta package in R for all outcomes. Feasibility will be assessed in terms of the number of data points, heterogeneity of treatment and outcome characteristics as well as the patient and study characteristics (Cipriani 2013). We will illustrate network geometry using network plots. If we identify relevant interventions that we are not aware of at the Protocol stage, we will consider them as eligible and we will include them in the network after assessing their comparability with the prespecified set of competing interventions. They will be included in the meta-analysis so long as they fulfil the transitivity assumptions as follow: that they are in principle jointly randomisable along with the other included interventions (that is, they have the same indication and could be meaningfully compared head-to-head with one another). We will also include contour-adjusted funnel plots for our primary outcomes to assess the potential impact of small studies on findings, if there are 10 or more included studies in these analyses.

\section{Subgroup analysis and investigation of heterogeneity}

If we find important heterogeneity or/and inconsistency (see above), we will explore the possible sources. If sufficient studies are available, we will also perform an NMA with meta-regression analyses on the primary outcome in Stata (Stata) for the following factors.

- Study setting (primary or community).
- Type of depression (first-episode; recurrent; chronic), as defined by the authors.

- Age of participants ( $<65$ years and $>65$ years).

- Comorbid anxiety (presence or absence, using an appropriate validated instrument, e.g. GAD-7).

- Length and number of sessions for psychological interventions.

- Funded by pharmaceutical company (yes or no).

\section{Sensitivity analysis}

We will undertake sensitivity analyses for the primary outcomes to investigate how effect estimates are affected by the following.

- Definition of relapse/recurrence: we will assess effects only in recurrent depression (i.e. exclude studies focusing on a single episode of depression).

- Drug classes: as there may be heterogeneity within drug classes we will also conduct NMAs including individual drugs as nodes.

- Dose: our primary analyses propose to include different doses as separate nodes in the network, we will assess the impact of combining doses of the same drug as a single node.

- Study bias: we will perform a sensitivity analysis excluding all studies assessed to be at high overall risk of bias and comparing the results.

- Provided there is sufficient information in the primary studies, we will compare relapse-recurrence rates in those studies that adequately controlled for withdrawal confounding (those that continued acute phase pharmacological management or those that documented this was weaned appropriately (NICE 2009)), versus those that did not (i.e. where ADM was stopped abruptly prior to the relapse prevention intervention, or where the approach was not documented)).

- Missing data: we will conduct analyses including analyses with OC data in addition to LOCF and other imputed data.

\section{Summary of findings and assessment of the certainty of the evidence}

We will present 'Summary of findings' tables, based on the template provided in Table 2 in Yepes-Nunes 2019 (but we will not report a column for ranking and will include a column for comments), for the following primary outcomes:

\section{Time to relapse-recurrence (within 12 months) \\ 2. Adverse outcomes (at any timepoint)}

The 'Summary of findings' tables will include all interventions.

For the relapse-recurrence related outcomes (time to relapserecurrence or binary outcome), we will assess our certainty in the evidence from the NMA using the Confidence In Network Meta-Analysis (CINeMA) approach (CINeMA; Nikolakopoulou 2020; Papakonstantinou 2020). CINeMA is based on the GRADE framework for assessing the certainty of the body of evidence and involves assessing the following six domains: within-study bias (i.e. the impact of risk of bias of the included trials); reporting bias (i.e. the impact of missing studies, outcomes and results); indirectness; imprecision; heterogeneity; and incoherence. Each domain (except 'reporting bias') is judged to have no concerns, some concerns or major concerns. 'Reporting bias' is judged as 'suspected' or 'undetected'. Judgements across the six domains are summarised to obtain four levels of confidence for each relative treatment effect: 
very low, low, moderate or high. We will assess our certainty in all comparisons formed by treatment groups in the decision and supplementary sets. Here we provide some further detail of the criteria we will use to inform our judgements for the domains.

\section{Within-study bias}

This domain concerns risk of bias within the included studies. In judging the certainty of the evidence for each relative effect estimate in this domain, we will consider the percentage contribution from studies judged to be at a low risk of bias, with some concerns, or a high risk of bias. Specifically, we will calculate a weighted average of the risk of bias, where the risk of bias judgements are assigned scores of -1 (low), 0 (some concerns) and 1 (high), and these scores are weighted by the proportion contributed from studies at each level. We will use these weighted averages to classify the certainty of evidence for each estimate. We may choose to override some of the judgements based on the findings from our sensitivity analysis that excludes trials at a high risk of bias.

\section{Indirectness}

In judging the certainty of the evidence for each relative effect estimate in this domain, we will consider for each study how directly it addresses the research question in combination with the percentage contribution the study makes to the estimate. In considering directness, we will consider population, intervention and outcome characteristics that are potential effect modifiers (e.g. age).

\section{Reporting bias}

We will use the results from our investigation of reporting bias (see 'Assessment of reporting biases', above) in judging the certainty of evidence for each relative effect estimate. This will involve considering the contribution that each direct comparison makes to each relative effect estimate (i.e. network estimate).

\section{Imprecision}

In judging the certainty of the evidence for each relative effect estimate in this domain, we have set a "margin of equivalence" for relapse-recurrence outcomes. The margin of equivalence corresponds to clinically unimportant differences between groups. Our margin of equivalence for relapse-recurrence outcomes corresponds to a risk ratio of between 0.8 and 1.25. We have derived this by assuming a comparator risk of 0.5 , based on expected baseline relapse rates, and a risk difference of 0.1 , based on agreement among the research team that a $10 \%$ improvement would be clinically meaningful in this cohort.

\section{Heterogeneity}

We will use the clinical equivalence ranges specified for the 'imprecision' domain in combination with prediction intervals, to determine whether heterogeneity in the results affects our certainty in the evidence.

\section{Incoherence}

We will take into account both global and local approaches to inconsistency. For local approaches, we will use the clinical equivalence ranges specified for the 'imprecision' domain in combination with the $95 \% \mathrm{Cls}$ of direct and indirect relative treatment effects to determine if incoherence between these estimates affects our certainty in the evidence.

\section{ACKNOWLEDGEMENTS}

We thank the editorial team of the Cochrane Common Mental Disorders (CCMD) Group including Jessica Hendon (Managing Editor) and Sarah Dawson (Information Specialist). Sarah Dawson helped develop the search strategies.

The authors and the CCMD Editorial Team are grateful to the following peer reviewers for their time and comments: Mieke van Driel, Ellen van Leeuwen, Jean Sellar-Edmunds and Gill Worthy. They would also like to thank Cochrane Copy Edit Support for the team's help.

The National Institute for Health Research (NIHR) is the largest single funder of the CCMD Group. Andrew Moriarty is funded by a NIHR Doctoral Research Fellowship for this research project (NIHR Doctoral Research Fellowship, Dr Andrew Moriarty, DRF-2018-11ST2-044). This publication presents independent research funded by the NIHR. The views expressed are those of the authors and not necessarily those of the NHS, the NIHR or the Department of Health and Social Care. 


\section{RE F E R E N C E S}

\section{Additional references}

\section{Ali 2017}

Ali S, Rhodes L, Moreea O, McMillan D, Gilbody S, Leach C, et al. How durable is the effect of low intensity CBT for depression and anxiety? Remission and relapse in a longitudinal cohort study. Behaviour Research and Therapy 2017;94:1-8.

\section{Aluko 2020}

Aluko P, Graybill E, Craig D, Henderson C, Drummond M, Wilson ECF, et al, on behalf of the Campbell and Cochrane Economics Methods Group. Chapter 20: Economic evidence. In: Higgins JPT, Thomas J, Chandler J, Cumpston M, Li T, Page MJ, Welch VA (editors). Cochrane Handbook for Systematic Reviews of Interventions version 6.1 (updated September 2020). Cochrane, 2020. Available from www.training.cochrane.org/ handbook.

\section{Beshai 2011}

Beshai S, Dobson KS, Bockting CLH, Quigley L. Relapse and recurrence prevention in depression: current research and future prospects. Clinical Psychology Review 2011;31(8):1349-60.

\section{Bockting 2015}

Bockting CL, Hollon SD, Jarrett RB, Kuyken W, Dobson K. A lifetime approach to major depressive disorder: the contributions of psychological interventions in preventing relapse and recurrence. Clinical Psychology Review 2015;41:16-26.

\section{Buckman 2018}

Buckman JEJ, Underwood A, Clarke K, Saunders R, Hollon SD, Fearon $P$, et al. Risk factors for relapse and recurrence of depression in adults and how they operate: a four-phase systematic review and meta-synthesis. Clinical Psychology Review 2018;64:13-38.

\section{Chaimani 2012}

Chaimani A, Salanti G. Using network meta-analysis to evaluate the existence of small-study effects in a network of interventions. Research Synthesis Methods 2012;3(2):161-76.

\section{Chaimani 2020}

Chaimani A, Caldwell DM, Li T, Higgins JPT, Salanti G. Chapter 11: Undertaking network meta-analyses. In: Higgins JPT, Thomas J, Chandler J, Cumpston M, Li T, Page MJ, Welch VA (editors). Cochrane Handbook for Systematic Reviews of Interventions version 6.1 (updated September 2020), Cochrane, 2020. Available from www.training.cochrane.org/handbook.

\section{CINeMA [Computer program]}

Institute of Social and Preventive Medicine, University of Bern CINeMA: Confidence in Network Meta-Analysis. Bern: Institute of Social and Preventive Medicine, University of Bern, 2017. Available from cinema.ispm.ch.

\section{Cipriani 2013}

Cipriani A, Higgins JPT, Geddes JR, Salanti G. Conceptual and technical challenges in network meta-analysis. Annals of Internal Medicine 2013;159(2):130-7.

\section{Clark 2011}

Clark DM. Implementing NICE guidelines for the psychological treatment of depression and anxiety disorders: the IAPT experience. International Review of Psychiatry 2011;23(4):31827.

\section{Clarke 2015}

Clarke K, Mayo-Wilson E, Kenny J, Pilling S. Can nonpharmacological interventions prevent relapse in adults who have recovered from depression? A systematic review and meta-analysis of randomised controlled trials. Clinical Psychology Review 2015;39:58-70.

\section{Cohen 2010}

Cohen D, Recalt A. Discontinuing Psychotropic Drugs from Participants in Randomized Controlled Trials: A Systematic Review. Psychother Psychosom 2019;88(2):96-104.

\section{Coupland 2011}

Coupland C, Dhiman P, Morriss R, Arthur A, Barton G, Hippisley-Cox J. Antidepressant use and risk of adverse outcomes in older people: population based cohort study. BMJ 2011;343(7819):1-15.

\section{Cox 2012}

Cox GR, Fisher CA, De Silva S, Phelan M, Akinwale OP, Simmons $M B$, et al. Interventions for preventing relapse and recurrence of a depressive disorder in children and adolescents. Cochrane Database of Systematic Reviews 2012, Issue 11. Art. No: CD007504. [DOI: 10.1002/14651858.CD007504.pub2]

\section{Cuijpers 2013}

Cuijpers P, Sijbrandij M, Koole SL, Andersson G, Beekman AT, Reynolds CF. The efficacy of psychotherapy and pharmacotherapy in treating depressive and anxiety disorders: a meta-analysis of direct comparisons. World Psychiatry 2013;12(2):137-48.

\section{Davies 2019}

Davies J, Read J. A systematic review into the incidence, severity and duration of antidepressant withdrawal effects: are guidelines evidence-based? Addictive Behaviours 2019;97:111-21.

\section{Deeks 2020}

Deeks JJ, Higgins JPT, Altman DG (editors). Chapter 10: Analysing data and undertaking meta-analyses. In: Higgins JPT, Thomas J, Chandler J, Cumpston M, Li T, Page MJ, Welch VA (editors). Cochrane Handbook for Systematic Reviews of Interventions version 6.1. The Cochrane Collaboration, 2020.

\section{Efthimiou 2016}

Efthimiou O, Debray TPA, van Valkenhoef G, Trelle S, Panayidou K, Moons KGM, et al. GetReal in network meta- 
analysis: a review of the methodology. Research Synthesis Methods 2016;7(3):236-63.

\section{Frank 1991}

Frank E, Prien RF, Jarrett RB, Keller MB, Kupfer DJ, Lavori PW, et al. Conceptualization and rationale for consensus definitions of terms in major depressive disorder: remission, recovery, relapse, and recurrence. JAMA Psychiatry 1991;48(9):851-5.

\section{Furukawa 2005}

Furukawa TA, Cipriani A, Barbui C, Brambilla P, Watanabe N. Imputing response rates from means and standard deviations in meta-analyses. International Clinical Psychopharmacology 2005;20(1):49-52.

\section{Furukawa 2006}

Furukawa TA, Barbui C, Cipriani A, Brambilla P, Watanabe N. Imputing missing standard deviations in meta-analyses can provide accurate results. Journal of Clinical Epidemiology 2006;59(1):7-10

\section{Gauthier 2019}

Gauthier G, Mucha L, Shi S, Guerin A. Economic burden of relapse/recurrence in patients with major depressive disorder. Journal of Drug Assessment 2019;8(1):97-103.

\section{GBD 2017}

Global Burden of Disease 2017 Disease and Injury Incidence and Prevalence Collaborators. Global, regional, and national incidence, prevalence, and years lived with disability for 354 diseases and injuries for 195 countries and territories, 19902017: a systematic analysis for the Global Burden of Disease Study 2017. The Lancet 2018;392(10159):1789-1858.

\section{Geddes 2003}

Geddes JR, Carney SM, Davies C, Furukawa TA, Kupfer DJ, Frank E, et al. Relapse prevention with antidepressant drug treatment in depressive disorders: a systematic review. Lancet 2003;361(9358):653-61.

\section{Gili 2015}

Gili M, Vicens C, Roca M, Andersen P, McMillan D. Interventions for preventing relapse or recurrence of depression in primary health care settings: a systematic review. Preventive Medicine 2015;76(S):S16-21.

\section{Glue 2010}

Glue P, Donovan MR, Kolluri S, Emir B. Meta-Analysis of Relapse Prevention Antidepressant Trials in Depressive Disorders. Australian and New Zealand Journal of Psychiatry 2010;44(8):697-705.

\section{Gunn 2013}

Gunn J, Elliott P, Densley K, Middleton A, Ambresin G, Dowrick C, et al. A trajectory-based approach to understand the factors associated with persistent depressive symptoms in primary care. Journal of Affective Disorders 2013;148(2-3):338-46.

\section{Hansen 2008}

Hansen R, Gaynes B, Thieda P, Gartlehner G, DeveaughGeiss A, Krebs E, et al. Meta-analysis of major depressive disorder relapse and recurrence with second-generation antidepressants. Psychiatric Services 2008;59(10):1121-30.

\section{Healy 1997}

Healy, D. The antidepressant era. Harvard University Press, 1997.

\section{Hengartner 2020}

Hengartner MP. How effective are antidepressants for depression over the long term? A critical review of relapse prevention trials and the issue of withdrawal confounding. Therapeutic Advances in Psychopharmacology 2020;10:2045125320921694.

\section{Higgins 2012}

Higgins JPT, Jackson D, Barrett JK, Lu G, Ades AE, White IR. Consistency and inconsistency in network meta-analysis: concepts and models for multi-arm studies. Research Synthesis Methods 2012;3(2):98-110.

\section{Higgins 2020}

Higgins JPT, Thomas J, Chandler J, Cumpston M, Li T, Page MJ, Welch VA (editors). Cochrane Handbook for Systematic Reviews of Interventions version 6.1 (updated September 2020). Cochrane, 2020. Available from www.training.cochrane.org/ handbook.

\section{Higgins 2021}

Higgins JPT, Savović J, Page MJ, Elbers RG, Sterne JAC. Chapter 8: Assessing risk of bias in a randomized trial. In: Higgins JPT, Thomas J, Chandler J, Cumpston M, Li T, Page MJ, Welch VA (editors). Cochrane Handbook for Systematic Reviews of Interventions version 6.2 (updated February 2021). Cochrane, 2021. Available from training.cochrane.org/handbook.

\section{Insel 2009}

Insel TR. Translating scientific opportunity into public health impact. Archives of General Psychiatry 2009;66(2):128.

\section{Kamenov 2016}

Kamenov K, Twomey C, Cabello M, Prina AM, Ayuso-Mateos JL. The efficacy of psychotherapy, pharmacotherapy and their combination on functioning and quality of life in depression: a meta-analysis. Psychological Medicine 2016;47(3):414-25.

\section{Kato 2020}

Kato $\mathrm{M}$, Hori $\mathrm{H}$, Inoue $\mathrm{T}$, Iga J, Iwata $\mathrm{M}$, Inagaki $\mathrm{T}$, Shinohara K, Imai H, Murata A, Mishima K, Tajika A. Discontinuation of antidepressants after remission with antidepressant medication in major depressive disorder: a systematic review and meta-analysis. Molecular Psychiatry 2020;26:118-133.

\section{Kaymaz 2008}

Kaymaz N, van Os J, Loonen AJ, Nolen WA. Evidence that patients with single versus recurrent depressive episodes are differentially sensitive to treatment discontinuation: a metaanalysis of placebo-controlled randomized trials. Journal of Clinical Psychiatry 2008;69(9):1423-36. 


\section{Kuyken 2015}

Kuyken W, Hayes R, Barrett B, Byng R, Dalgleish T, Kessler D, . Effectiveness and cost-effectiveness of mindfulness-based cognitive therapy compared with maintenance antidepressant treatment in the prevention of depressive relapse or recurrence (PREVENT): a randomised controlled trial. Lancet 2015;386(9988):63-73.

\section{Lam 2009}

Lam RW, Kennedy SH, Grigoriadis S, Mclntyre RS, Milev R, Ramasubbu R, et al. Canadian Network for Mood and Anxiety Treatments (CANMAT) Clinical guidelines for the management of major depressive disorder in adults. III. Pharmacotherapy. Journal of Affective Disorders 2009;117:S26-43.

\section{Lenora 2019}

Lenora R, Kumar A, Uphoff E, Meader N, Furtado VA. Interventions for helping people recognise early signs of recurrence in depression. Cochrane Database of Systematic Reviews 2019, Issue 7. Art. No: CD013383. [DOI: 10.1002/14651858.CD013383]

\section{Lin 1998}

Lin EHB, Katon WJ, VonKorff M, Russo JE, Simon GE, Bush TM, Ludman E. Relapse of depression in primary care: rate and clinical predictors. Archives of Family Medicine 1998;7(5):443-9.

\section{Machmutow 2019}

Machmutow K, Meister R, Jansen A, Kriston L, Watzke B, Härter MC, et al. Comparative effectiveness of continuation and maintenance treatments for persistent depressive disorder in adults. Cochrane Database of Systematic Reviews 2019, Issue 5. Art. No: CD012855. [DOI: 10.1002/14651858.CD012855.pub2]

\section{Maund 2019}

Maund E, Dewar-Haggart R, Williams S, Bowers $\mathrm{H}$, Geraghty AWA, Leydon G, et al. Barriers and facilitators to discontinuing antidepressant use: a systematic review and thematic synthesis. Journal of Affective Disorders 2019;245:38-62.

\section{Mavridis 2016}

Mavridis D, Efthimiou O, Leucht S, Salanti G. Publication bias and small-study effects magnified effectiveness of antipsychotics but their relative ranking remained invariant. Journal of Clinical Epidemiology 2016;69:161-9.

\section{Monroe 2011}

Monroe SM, Harkness KL. Recurrence in major depression: a conceptual analysis. Psychological Review 2011;118(4):655-74.

\section{NICE 2009}

National Institute for Health and Care Excellence. Depression in adults: recognition and management. NICE Guideline (CG 90) 2009.

\section{NICE 2018}

National Institute for Health and Care Excellence. Depression in adults: treatment and management. NICE guideline: short version (draft for second consultation) 2018:1-76.

\section{Nikolakopoulou 2020}

Nikolakopoulou A, Higgins JPT, Papakonstantinou T, Chaimani A, Giovane CD, Egger M, et al. Cinema: An approach for assessing confidence in the results of a network metaanalysis. PLoS Medicine 2020;17(4):1-19.

\section{Ormel 2020}

Ormel J, Spinhoven P, De Vries Y, Cramer A, Siegle G, Bockting C, Hollon S. The antidepressant standoff: Why it continues and how to resolve it. Psychological Medicine 2020;50(2):177-186.

\section{Page 2019}

Page MJ, Higgins JP, Sterne JA. Chapter 13: Assessing risk of bias due to missing results in a synthesis. In: Higgins JPT, Thomas J, Chandler J, Cumpston M, Li T, Page MJ, Welch VA (editors). Cochrane Handbook for Systematic Reviews of Interventions version 6.0 (updated July 2019). Cochrane, 2019. Available from www.training.cochrane.org/handbook.

\section{Papakonstantinou 2020}

Papakonstantinou T, Nikolakopoulou A, Higgins JPT, Egger M, Salanti G. CINeMA: Software for semiautomated assessment of the confidence in the results of network meta-analysis. Campbell Systematic Reviews 2020;16(1):1-15.

\section{$\mathbf{R}$ [Computer program]}

R Foundation for Statistical Computing R: A language and environment for statistical computing. Version 3.4.2. Vienna, Austria: R Foundation for Statistical Computing, 2017. Available at www.R-project.org.

\section{Rait 2009}

Rait G, Walters K, Griffin M, Buszewicz M, Petersen I, Nazareth I. Recent trends in the incidence of recorded depression in primary care. British Journal of Psychiatry 2009;195(6):520-4.

\section{Ramanuj 2019}

Ramanuj P, Ferenchick EK, Pincus HA. Depression in primary care: part 2-management. BMJ (Clinical Research Edition) 2019;365:1835.

\section{Recalt 2019}

Recalt AM, Cohen D. Withdrawal confounding in randomized controlled trials of antipsychotic, antidepressant, and stimulant drugs 2000-2017. Psychotherapy and Psychosomatics 2019;88(2):105-13.

\section{Review Manager 2020 [Computer program]}

The Cochrane Collaboration Review Manager (RevMan) Version 5.4. The Cochrane Collaboration, 2020.

\section{RevMan Web 2019 [Computer program]}

The Cochrane Collaboration Review Manager Web (RevMan Web). The Cochrane Collaboration, 2019. Available at revman.cochrane.org.

\section{Richards 2011}

Richards D. Prevalence and clinical course of depression: a review. Clinical Psychology Review 2011;31(7):1117-25. 


\section{Riso 1997}

Riso LP, Thase ME, Howland RH, Friedman ES, Simons AD, Tu XM. A prospective test of criteria for response, remission, relapse, recovery, and recurrence in depressed patients treated with cognitive behavior therapy. Journal of Affective Disorders 1997;43(2):131-42.

\section{Rucker 2012}

Rucker G. Network meta-analysis, electrical networks and graph theory. Research Synthesis Methods 2012;3:312-324.

\section{Rush 2006}

Rush AJ, Kraemer HC, Sackeim HA, Fava M, Trivedi MH, Frank E, et al. Report by the ACNP Task Force on response and remission in major depressive disorder. Neuropsychopharmacology 2006;31(9):1841-53.

\section{Solomon 2000}

Solomon DA, Keller MB, Leon AC, Mueller TI, Lavori PW, Shea MT, et al. Multiple recurrences of major depressive disorder. American Journal of Psychiatry 2000;157(2):229-33.

\section{Stata [Computer program]}

Stata. Version 15. College Station, TX, USA: StataCorp, 2017. Available from www.stata.com.

\section{Steinert 2014}

Steinert C, Hofmann M, Kruse J, Leichsenring F. The prospective long-term course of adult depression in general practice and the community. A systematic literature review. Journal of Affective Disorders 2014;152-154:65-75.

\section{Sterne 2019}

Sterne J A C, Savović J, Page M J, Elbers R G, Blencowe N S, Boutron I et al. RoB 2: a revised tool for assessing risk of bias in randomised trials. BMJ 2019;366:14898. [DOI: 10.1136/ bmj.14898]

\section{Tickell 2020}

Tickell A, Byng R, Crane C, Gradinger F, Hayes R, Robson J, et al. Recovery from recurrent depression with mindfulness-based cognitive therapy and antidepressants: a qualitative study with illustrative case studies. BMJ Open 2020;10(2):e033892.

\section{Van Kempen 2010}

Van Kempen GMJ, Pearlman T, Karasu TB, Merriam AE, Glassman AH, Gelenberg AJ, et al. Practice guideline for adult major depressive disorder [15]. American Journal of Psychiatry 2010;151(4):625-6.

\section{Van Leeuwen 2020}

Van Leeuwen E, van Driel ML, De Sutter AIM, Anderson K, Robertson L, Christiaens T. Discontinuation of long-term antidepressant use for depressive and anxiety disorders in adults. Cochrane Database of Systematic Reviews 2020;2:CD013495.

\section{Van Weel-Baumgarten 2000}

van Weel-Baumgarten, EM, Schers HJ, van den Bosch WJ, van den Hoogen HJ, Zitman FG. Long-term follow-up of depression among patients in the community and in family practice settings - a systematic review. Journal of Family Practice 2000;49(12):1113-1120.

\section{Vittengl 2007}

Vittengl J, Clark L, Dunn T, Jarrett R. Reducing relapse and recurrence in unipolar depression: a comparative meta-analysis of cognitive-behavioral therapy's effects. Journal of Consulting and Clinical Psychology 2007;75(3):475-88.

\section{WHO 2017}

World Health Organization. Depression and Other Common Mental Disorders: Global Health Estimates. Geneva 2017.

\section{Yepes-Nunes 2019}

Yepes-Nuñez JJ, Li S, Guyatt G, Jack SM, Brozek JL, Beyene J, et al. Development of the summary of findings table for network meta-analysis. Journal of Clinical Epidemiology 2019;115:1-13.

\section{APPENDICES}

\section{Appendix 1. MEDLINE search strategy}

MEDLINE-ALL (Ovid) <1946 onwards>

Search Strategy:

[Population]

1 *Depression/

2 Depressive Disorder/

3 Depressive Disorder, Major/

4 Depressive Disorder, Treatment-resistant/

5 depress ${ }^{\star}$. ti. 
6 (depress $^{\star} \operatorname{adj5}\left(\right.$ relaps $^{\star}$ or recurr* or maintenance or continuation or prophyla*)).ti,ab,kf,kw.

7 (depress $^{\star}$ adj5 (chronic ${ }^{\star}$ or clinical ${ }^{\star}$ or diagnos ${ }^{\star}$ or disorder ${ }^{\star}$ or major or unipolar or illness or resistan* or refractory or scale* or score ${ }^{\star}$ or adult ${ }^{\star}$ or patient $^{\star}$ or participant ${ }^{\star}$ or people or geriatric ${ }^{\star}$ or elder ${ }^{\star}$ or middle aged)).ab,kf,kw.

8 or $/ 1-7$

[Outcome]

\section{Recurrence/}

10 Secondary Prevention/ or Tertiary Prevention/

11 * Preventive Health Services/

12 ((prevent ${ }^{\star}$ or reduc $\left.{ }^{\star}\right)$ adj5 (future or repeat ${ }^{\star}$ or relaps $^{\star}$ or recurr $^{\star}$ or secondary or tertiary or target ${ }^{\star}$ or indicat $\left.\left.^{\star}\right)\right) . t i, a b, k f, k w$.

13 ((time or timing) adj2 (relaps ${ }^{\star}$ or recurr $\left.\left.{ }^{\star}\right)\right) . t i, a b, k f, k w$.

14 ((continuation or maintenance) adj2 (treatment* or therap* or psycotherap ${ }^{\star}$ or phase or antidepress ${ }^{\star}$ or anti-depress* or medicat $\left.\left.^{\star}\right)\right) . t i, a b, k f, k w$.

15 or/9-14

16 (8 and 15)

17 depress $^{\star}$.ti. and ((relapse or recurr ${ }^{\star}$ or maintenance or continuation or prophyla*) adj5 (recovered or remission or remit ${ }^{\star}$ or responder ${ }^{\star}$ or "responded to" or recent* or previous* or past)).ti,ab,kf,kw.

$18(16$ or 17$)$

[RCT Filter]

19 controlled clinical trial.pt.

20 randomized controlled trial.pt.

21 clinical trials as topic/

22 (randomi\#ed or randomi\#ation or randomi\#ing).ti,ab,kf.

23 (RCT or "at random" or (random* adj3 (administ* or allocat ${ }^{\star}$ or assign* or class ${ }^{\star}$ or cluster or crossover or cross-over or control* or determine $^{\star}$ or divide* or division or distribut ${ }^{\star}$ or expose ${ }^{\star}$ or fashion or number ${ }^{\star}$ or place* or pragmatic or quasi or recruit ${ }^{\star}$ or split or subsitut $^{\star}$ or treat $\left.\left.^{\star}\right)\right)$ ).ti,ab,kf,kw.

24 placebo.ab,ti,kf.

25 trial.ti.

26 (control $^{\star}$ adj3 group $\left.{ }^{\star}\right) \cdot a b$.

27 (control ${ }^{\star}$ and (trial or study or group ${ }^{\star}$ ) and (waitlist ${ }^{\star}$ or wait $^{\star}$ list $^{\star}$ or ((treatment or care) adj2 usual))).ti,ab,kf,kw,hw.

28 ((single or double or triple or treble) adj2 (blind* or mask* or dummy)).ti,ab,kf,kw.

29 double-blind method/ or random allocation/ or single-blind method/

30 or $/ 19-29$

31 exp animals/ not humans.sh.

$32(30$ not 31)

33 (18 and 32)

[Primary Care Filter]

34 Primary Health Care/ or "Continuity of Patient Care"/ or Patient Handoff/ or Transition to Adult Care/

Interventions for preventing relapse or recurrence of major depressive disorder in adults in a primary care setting: a network meta-

Copyright (c) 2021 The Cochrane Collaboration. Published by John Wiley \& Sons, Ltd. 
35 Physicians, Family/

36 General Practice/ or Family Practice/

37 *Family Health/

38 (primary adj2 (care or health*)).ti,ab,kf,kw.

39 (GP or "GP's" or generalist $\left.{ }^{\star}\right) . a b$.

40 ((general or family or nurs ${ }^{\star}$ ) adj1 (practice ${ }^{\star}$ or practitioner $\left.\left.{ }^{\star}\right)\right) . t i, a b, k f, k w$.

41 Nurse Practitioners/

42 Primary Care Nursing /

43 Family Nursing/

44 Home Nursing/

45 Community Health Centers/ or Community Mental Health Centers/

46 (homecare or home care or (care adj3 community)).ti,ab,kf,kw.

47 ((family or community or practice $\left.{ }^{\star}\right)$ adj $\left(\right.$ medic $^{\star}$ or doctor ${ }^{\star}$ or physician* or health or nurs $\left.\left.^{\star}\right)\right) . t i, a b, k f, k w$.

48 ((in or at or based or own) adj2 (home or homes)).ab.

49 Private Practice/

50 private practice ${ }^{\star} . \mathrm{ti}, \mathrm{ab}, \mathrm{kf}$.

51 Ambulatory Care/

52 (("Access to Psychological Therapies" or IAPT) or (psych* adj3 practitioner*)).ti,ab,kf,kw.

53 or/34-52

54 (33 and 53)

\section{Appendix 2. Glossary of terms}

Recovery: follows an extended period of remission; at this point, patients are said to be no longer in episode.

Recurrence: a new episode of depression, separate from the index episode.

Relapse: a return to the index episode of depression.

Relapse-recurrence: a hybrid term used to describe the re-emergence of depressive symptoms after a period of relative wellness, to account for inconsistencies in how relapse and recurrence are operationalised in the literature.

Remission: a period of time, following a response to treatment, during which patients have minimal depressive symptoms but are still "in episode".

Response: an initial improvement of symptoms, usually after treatment initiation and usually attributable to the treatment.

\section{CONTRIBUTIONSOF AUTHORS}

ASM: Contributed to the conception of the review and lead author of the protocol. Wrote the first draft of the protocol and response to peer review.

LR: Commented on the background and contributed to the methodology of the protocol.

FM: Commented on the background and contributed to the methodology of the protocol.

NC: Commented on the background and contributed to the methodology of the protocol.

SG: Contributed to the conception of the review and the background and methodology of the protocol.

DM: Contributed to the conception of the review and the background and methodology of the protocol.

CCG: Contributed to the conception of the review and the background and methodology of the protocol.

Interventions for preventing relapse or recurrence of major depressive disorder in adults in a primary care setting: a network meta- 
SA: Contributed to the conception of the review and the background and methodology of the protocol.

$\mathrm{SH}$ : Commented on the background and contributed to the methodology of the protocol.

$\mathrm{RC}$ : Commented on the background and contributed to the methodology of the protocol.

NM: Commented on the background and developed the methodology of the protocol.

\section{DECLARATIONS OF INTEREST}

ASM: No conflicts of interest.

LR: No conflicts of interest.

FM: No conflicts of interest.

NC: No conflicts of interest.

SG: No conflicts of interest.

DM: No conflicts of interest.

CCG: No conflicts of interest.

SA: No conflicts of interest.

$\mathrm{SH}$ : is the Joint Co-ordinating Editor of the Cochrane Common Mental Disorders Group. She is funded by the Auckland Medical Research Foundation and CureKids.

RC: Leads and has responsibility for Cochrane Common Mental Disorders, which has supported parts of the review process and is largely funded by a grant from the National Institute for Health Research (NIHR) in the UK.

NM: is the Deputy Co-ordinating Editor of the Cochrane Common Mental Disorders Group.

\section{SOURCES OF SUPPORT}

\section{Internal sources}

- University of York, UK

- Hull York Medical School, UK

- University of Auckland, New Zealand

\section{External sources}

- National Institute for Health Research (NIHR), UK

LR, RC \& NM contribution to this protocol is supported by Cochrane Infrastructure funding to the Common Mental Disorders Cochrane Review Group.

ASM is funded by a NIHR Doctoral Research Fellowship for this research project. This publication presents independent research funded by the NIHR. The views expressed are those of the authors and not necessarily those of the NHS, the NIHR or the Department of Health and Social Care. 